Communication

\title{
Influence of Carbohydrates on Secondary Metabolism in Fusarium avenaceum
}

\section{Jens Laurids Sørensen * and Henriette Giese}

Department of Biotechnology, Chemistry and Environmental Engineering, Aalborg University, Sohngaardsholmsvej 49, Aalborg DK-9000, Denmark; E-Mail: hgiese@bio.aau.dk

* Author to whom correspondence should be addressed; E-Mail: jls@bio.aau.dk; Tel.: +45-9940-8524; Fax: +45-9814-1808.

Received: 3 September 2013; in revised form: 16 September 2013 / Accepted: 16 September 2013 / Published: 24 September 2013

\begin{abstract}
Fusarium avenaceum is a widespread pathogen of important crops in the temperate climate zones that can produce many bioactive secondary metabolites, including moniliformin, fusarin C, antibiotic Y, 2-amino-14,16-dimethyloctadecan-3-ol (2-AOD-3-ol), chlamydosporol, aurofusarin and enniatins. Here, we examine the production of these secondary metabolites in response to cultivation on different carbon sources in order to gain insight into the regulation and production of secondary metabolites in $F$. avenaceum. Seven monosaccharides (arabinose, xylose, fructose, sorbose, galactose, mannose, glucose), five disaccharides (cellobiose, lactose, maltose, sucrose and trehalose) and three polysaccharides (dextrin, inulin and xylan) were used as substrates. Three $F$. avenaceum strains were used in the experiments. These were all able to grow and produce aurofusarin on the tested carbon sources. Moniliformin and enniatins were produced on all carbon types, except on lactose, which suggest a common conserved regulation mechanism. Differences in the strains was observed for production of fusarin C, 2-AOD-3-ol, chlamydosporol and antibiotic Y, which suggests that carbon source plays a role in the regulation of their biosynthesis.
\end{abstract}

Keywords: secondary metabolites; moniliformin; enniatin; carbon; regulation; activation; creA 


\section{Introduction}

Fusarium avenaceum is among the most frequent fungal species encountered on small grain cereals and maize in temperate climate zones such as Northern Europe and Canada [1-5]. The species are pathogenic to diverse plants ranging from apples [6], rutabaga [7], potato tubers [8] and mung beans [9]. $F$. avenaceum is able to produce several bioactive secondary metabolites including moniliformin, fusarin C, antibiotic Y, 2-amino-14,16-dimethyloctadecan-3-ol (2-AOD-3-ol), chlamydosporol, aurofusarin and enniatins $[6,10]$.

Figure 1. Structures of secondary metabolites produced by $F$. avenaceum.
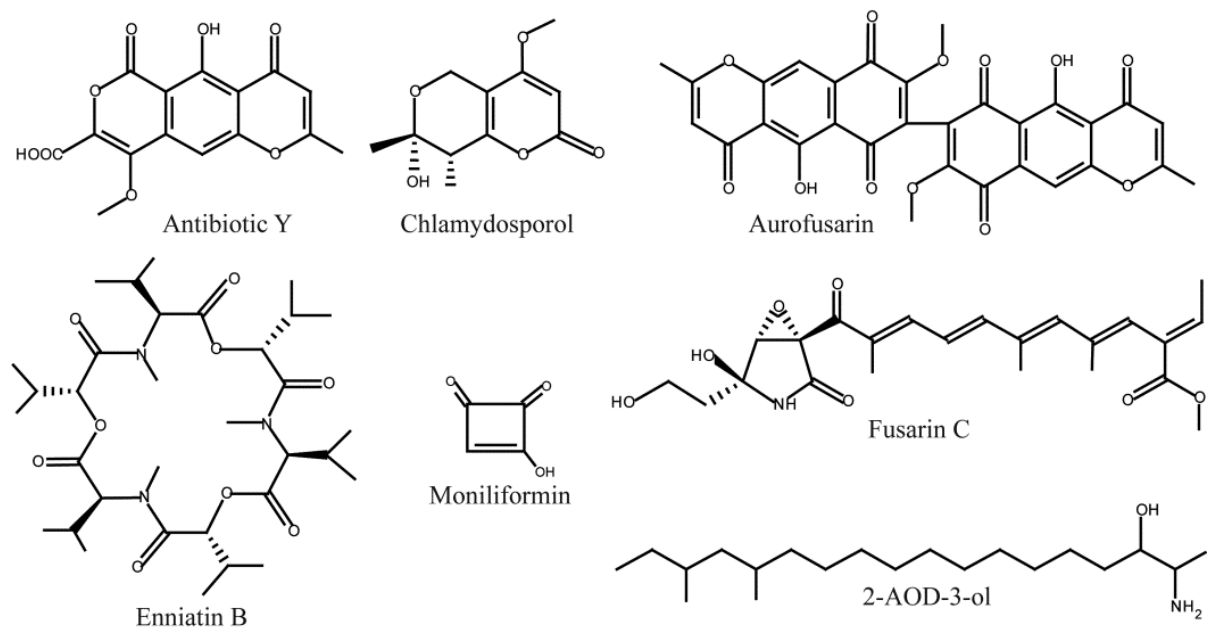

Several of these attract attention due to their presence in food and feed sources and their potential harmful effects on human and animal health. Moniliformin and enniatins have been characterized as emerging mycotoxins [11,12]. Some of the compounds co-occur in infected plants $[6,13]$, but little is known about the regulatory patterns which control production of secondary metabolites in $F$. avenaceum. Production of secondary metabolites is influenced by global regulators which directly or indirectly activate the respective genes or gene clusters [14]. The global regulators respond to a variety of abiotic components including $\mathrm{pH}$, light, temperature, water activity and nutrient availability [15]. Carbon and nitrogen sources are known to influence production of the trichothecene deoxynivalenol and the polyketide fusarielin $\mathrm{H}$ in $F$. graminearum [16-18]. AreA, the global nitrogen regulator has been shown to affect trichothecene production in F. graminearum [19] and bikaverin and gibberellins in F. fujikuroi [20,21]. A link between AreA and the global carbon catabolite repressor, CreA, has been found in Aspergillus nidulans with regard to arginine catabolism [22] and lactose has been used as a substrate for penicillin production in Penicillium chrysogenum [23]. There is little information on how CreA influence secondary metabolite production in fungi. In $F$. avenaceum water activity and temperature has been shown to have a limited influence on production of antibiotic $Y$, moniliformin and enniatins [24]. As F. avenaceum is able to colonize a diverse range of plants and tissues with different carbon composition we want to gain information on how production of known secondary metabolites in this fungal species is influenced by carbon source. 


\section{Results and Discussion}

\subsection{Quantification of Secondary Metabolites}

A LC-MS/MS method was developed for quantification of moniliformin, fusarin $\mathrm{C}$, antibiotic $\mathrm{Y}$, 2-AOD-3-ol, chlamydosporol, aurofusarin and enniatins. In order to obtain the optimal quantification conditions the MS/MS parameters and settings were automatically adjusted for each compound (Table 1 and Figure 2).

Table 1. Parameters for selected reaction monitoring (SRM) of $F$. avenaceum secondary metabolites.

\begin{tabular}{|c|c|c|c|c|c|}
\hline Compound & $\mathrm{RT}(\min )^{\mathrm{a}}$ & Precursor ion $(\mathrm{m} / \mathrm{z})$ & Product ions $(\mathrm{m} / \mathrm{z})^{\mathrm{b}}$ & S-lens $(\mathrm{eV})$ & $\operatorname{CID}(\mathrm{eV})^{\mathrm{c}}$ \\
\hline Moniliformin & 0.75 & $97.0[\mathrm{M}-\mathrm{H}]^{-}$ & 41.1 & 38 & 18 \\
\hline Chlamydosporol & 2.65 & $227.1[\mathrm{M}+\mathrm{H}]^{+}$ & $167.7 / 77.1$ & 63 & $36 / 15$ \\
\hline Antibiotic Y & 4.81 & $319.1[\mathrm{M}+\mathrm{H}]^{+}$ & $287.1 / 175.1$ & 79 & $22 / 30$ \\
\hline Fusarin C & $5.38-5.54$ & $432.2[\mathrm{M}+\mathrm{H}]^{+}$ & $185.1 / 213.2$ & 64 & $29 / 25$ \\
\hline Aurofusarin & 6.19 & $571.1[\mathrm{M}+\mathrm{H}]^{+}$ & $556.2 / 485.2$ & 200 & $34 / 24$ \\
\hline 2-AOD-3-ol & 6.36 & $314.4[\mathrm{M}+\mathrm{H}]^{+}$ & $69.1 / 97.1$ & 76 & $20 / 27$ \\
\hline Enniatin B & 7.67 & $657.5\left[\mathrm{M}+\mathrm{NH}_{4}\right]^{+}$ & $196.2 / 214.2$ & 136 & $30 / 31$ \\
\hline Enniatin B1 & 7.87 & $671.5\left[\mathrm{M}+\mathrm{NH}_{4}\right]^{+}$ & $196.2 / 214.2$ & 110 & $30 / 24$ \\
\hline Enniatin A1 & 8.10 & $685.5\left[\mathrm{M}+\mathrm{NH}_{4}\right]^{+}$ & $210.1 / 228.2$ & 113 & $30 / 30$ \\
\hline Enniatin A & 8.21 & $699.5\left[\mathrm{M}+\mathrm{NH}_{4}\right]^{+}$ & $210.1 / 228.2$ & 115 & $24 / 25$ \\
\hline
\end{tabular}

Notes: ${ }^{a}$ : Retention time; ${ }^{\mathrm{b}}$ : Quantifier/qualifier ions; ${ }^{\mathrm{c}}$ : Collision induced dissociation energy for quantifier/qualifier ions.

Although aurofusarin, chlamydosporol and 2-AOD-3-ol detections were adjusted based on a fungal extract we were able to quantify these secondary metabolites specifically without interference from other contaminating compounds. With the fusarin $\mathrm{C}$ settings we detected 2-3 peaks in all $F$. avenaceum extracts suggesting the presence of compounds with similar mass and fragmentation pattern such as the (7Z)- and (5Z)-fusarin $\mathrm{C}$ isoforms. This has previously been observed by a LC-MS/MS method specifically developed for fusarin C [25]. Two overlapping peaks were detected for chlamydosporol probably originating from the minor and major chlamydosporol epimers [26].

Figure 2. LC-MS/MS detection of quantification traces for moniliformin, fusarin $\mathrm{C}$, antibiotic Y, (2-AOD-3-ol), chlamydosporol, aurofusarin and enniatin B (ion count) produced by $F$. avenaceum (IBT 40847) grown on mannose.

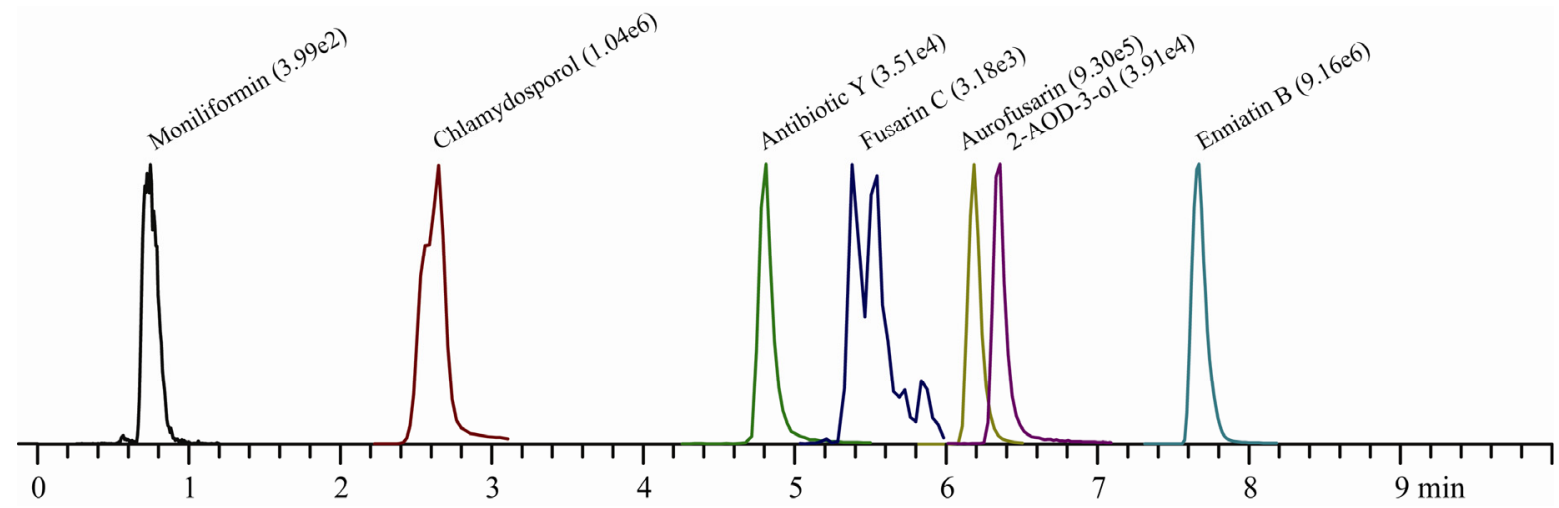




\subsection{Growth and Production of Secondary Metabolites}

Ergosterol levels were measured to quantify fungal growth of the three $F$. avenaceum strains on seven monosaccharides (arabinose, xylose, fructose, sorbose, galactose, mannose and glucose), five disaccharides (cellobiose, lactose, maltose, sucrose and trehalose) and three polysaccharides (dextrin, inulin and xylan). Glucose and fructose are predicted to act as repressors of cre A and arabinose as a derepressor [27]. Lactose is reported to be a poor carbon source for fungal growth and to act as a derepressor of $\operatorname{cre} A$ [28]. The three strains were able to utilize all carbon sources and growth was recorded visually and by ergosterol determination of all samples. The Ergosterol measurements showed that $F$. avenaceum strains IBT 8500 and IBT 40847 had similar growth patterns and generally grew better on the three polysaccharides than the mono- and disaccharides. Conversely strain IBT 5001 had slightly higher ergosterol levels when grown on mono- and disaccharides.

Figure 3. Production of secondary metabolites of the three $F$. avenaceum strains growing on different carbon sources. Error bars indicate standard error of mean of five replicates. Ergosterol, aurofusarin, 2-AOD-3-ol and chlamydosporols were quantified relatively and the maximum mean was set to $100 \%$.
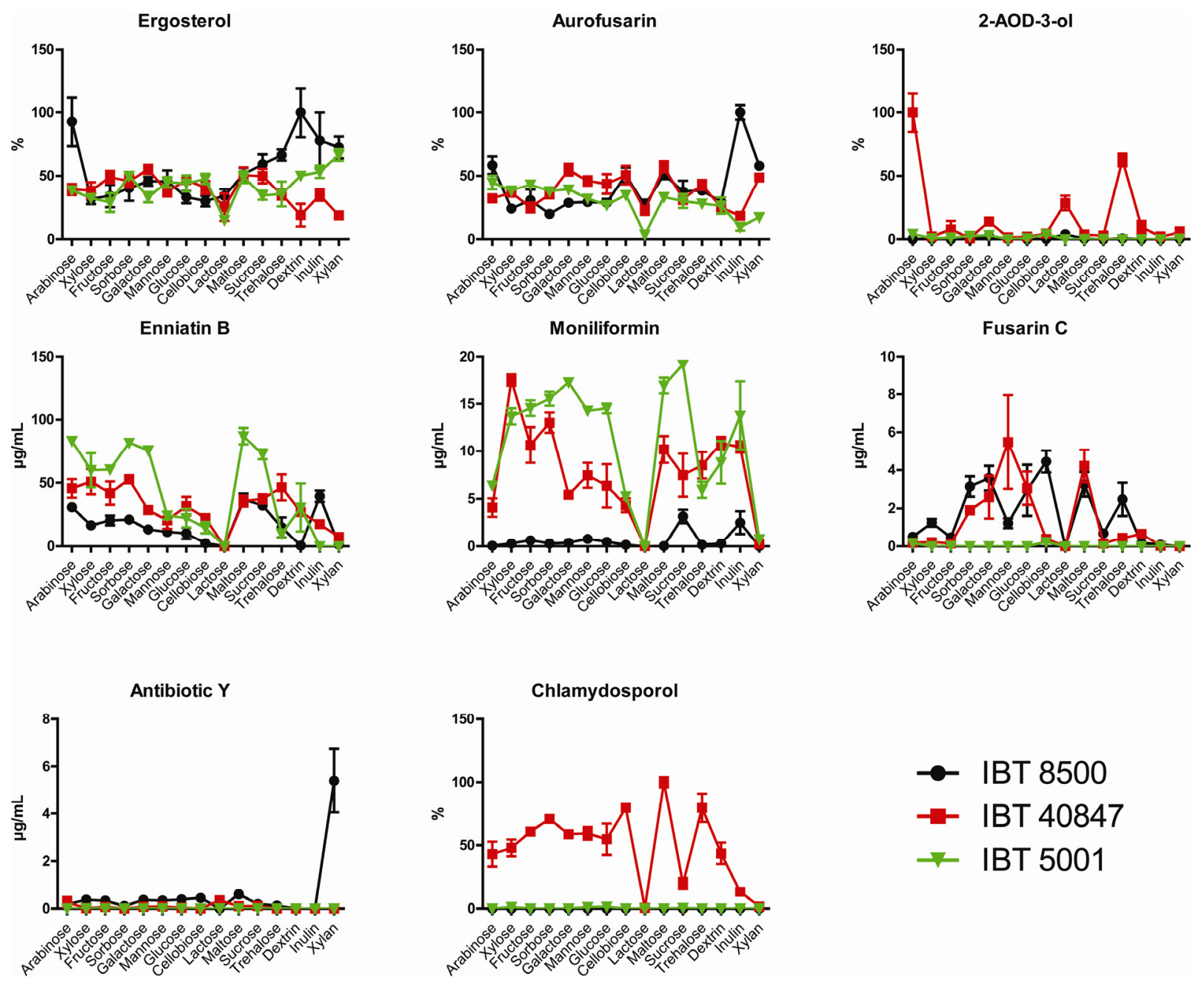

The developed LC-MS/MS method was used to measure production of secondary metabolites by three $F$. avenaceum strains in response to cultivation on the different carbon sources. The three strains 
exhibited some variations in preferred carbon source for production of the secondary metabolites. Aurofusarin was produced on all carbon sources by the three strains, where the highest levels were observed on inulin for IBT 8500, maltose for IBT 40847 and arabinose for IBT 5001. Lactose was, however, the poorest carbon source for aurofusarin production for all three strains. Strains IBT8500 and IBT 5001 only produced trace amounts of 2-AOD-3-olcompared to IBT 40847. Production of 2-AOD-3-ol in IBT 40847 seemed to be dependent on carbon source with mannose as the best substrate. Enniatin were produced in successively decreasing amounts in the sequence $\mathrm{B}>\mathrm{B} 1>\mathrm{A} 1>\mathrm{A}$, as observed previously [6], and therefore only enniatin B levels are shown. Production of enniatin B was similar in IBT 8500 and IBT 40847 where maltose, sucrose and inulin resulted in high levels. Enniatin B was produced in high levels on maltose and sucrose as well as on trehalose, xylose and sorbose by strain IBT 40847. Lactose was a poor substrate for enniatin B and moniliformin production in all strains. The best carbon source for moniliformin production was sucrose in IBT 8500 and IBT 5001, whereas IBT 40847 had highest production when grown on xylose. Cellobiose resulted in the highest levels of fusarin C in IBT 8500 and 5001, while it was mannose for IBT 40847. Antibiotic Y was not produced by IBT 5001 and only sparsely by IB T 40847 ( $\max 0.35 \mu \mathrm{g} / \mathrm{mL}$ ) under the tested nutrient conditions and lactose and arabinose appeared to be the best carbon source. Production of antibiotic Y was highly induced on xylan in IBT 8500 compared to the other carbon sources. Here, only low levels were observed as was the case for the two other strains under all the tested conditions. IBT 8500 did not produce chlamydosporol in these experiments and this compound was only detected in small amounts in IBT 5001 on four carbon sources (xylan, mannose, glucose and sucrose). IBT 40847 was a strong producer of chlamydosporol and produced it on all carbon sources, except lactose. The production of enniatin B and moniliformin followed a similar pattern in all three strains, which could suggest that they are regulated by the same mechanisms through a conserved pathway in $F$. avenaceum that is independent on creA. This means that moniliformin and enniatin are likely to co-occur in plants infected with $F$. avenaceum. In contrast to this the strains differed in the production of fusarin $\mathrm{C}$, antibiotic $\mathrm{Y}$ and chlamydosporol suggesting that regulation has evolved separately in the three strains. Variation between strains of the same species in production of secondary metabolites in response to different carbon sources was also observed for DON production in F. graminearum [17], which illustrates the need to include several strains when examining the effects of nutrients on secondary metabolism in a species.

Lactose provided the poorest source of carbon for production of secondary metabolites in the tested strains as aurofusarin, chlamydosporol, enniatin $\mathrm{B}$, moniliformin and fusarin $\mathrm{C}$ were all produced in low amounts. Lactose has previously been shown to stimulate production of lovastatin in Aspergillus terreus [29] and have been used as substrate for production of penicillin [23]. The negative effect of lactose on secondary metabolism in $F$. avenaceum observed in the present study is therefore not common feature for all fungi. Lactose is not a natural substrate for plant pathogenic fungi like $F$. avenaceum and the utilization of this carbon source may differ from fungi that are adapted to this substrate. However, little information is currently available on how individual mono-, di- and polysaccharides affect secondary metabolism and which mechanisms are involved. Carbon clearly has an effect on secondary metabolism in filamentous fungi but the regulatory pathways are probably interlinked with global regulators. 


\section{Experimental Section}

\subsection{Chemicals}

All chemical solvents were obtained from Thermo Fisher Scientific (San José, CA, USA) and carbon sources were purchased from Sigma-Aldrich (St. Louis, MO, USA) and Merck (Whitehouse Station, NJ, USA). Reference standards of enniatin mix (17\% A, $34 \%$ A1, 24\% B and 26\% B1) and antibiotic Y were purchased from Bioaustralis (Smithfield, Australia), moniliformin from Sigma-Aldrich, whereas fusarin $\mathrm{C}$ was available from previous studies [30].

\subsection{Fungal Isolates and Cultivation}

Three $F$. avenaceum strains (IBT 8500, 40847 and 5001) were selected from the IBT collection at the Technical University of Denmark. Conidia were produced by growing the strains in a $250 \mathrm{~mL}$ baffled flasks containing $50 \mathrm{~mL}$ liquid sporulation medium [31] for three days at $20{ }^{\circ} \mathrm{C}$ in the dark at $150 \mathrm{rpm}$. The spores were isolated by centrifugation and dissolved in sterile $\mathrm{H}_{2} \mathrm{O}$ to give a final concentration of $1 \times 10^{6}$ spores per mL. Cultivation on different carbon sources was performed as previously described [18]. In brief, twenty $\mu \mathrm{L}$ spore suspension was inoculated to $14 \mathrm{~mL}$ culture tubes containing $1 \mathrm{~mL}$ modified czapek dox (CZ) medium ( $\mathrm{pH}$ 6) containing: $30 \mathrm{mg}$ carbon source (L-arabinose, D-xylose, D-fructose, L-sorbose, D-galactose, D-mannose, D-glucose, D-cellobiose, $\beta$-lactose, maltose, sucrose, dextrin from corn, D-xylan and D-trehalose); $1.5 \mathrm{mg}$ arginine; $1 \mathrm{mg} \mathrm{K}_{2} \mathrm{HPO}_{4}$; $0.5 \mathrm{mg} \mathrm{KCl} ; 0.5 \mathrm{mg} \mathrm{MgSO} \cdot 7 \mathrm{H}_{2} \mathrm{O} ; 0.01 \mathrm{mg} \mathrm{FeSO} \cdot 7 \mathrm{H}_{2} \mathrm{O}$ and $1 \mu \mathrm{L}$ trace solution $\left(1 \mathrm{~g} \mathrm{ZnSO} \mathrm{Zn}_{4} \cdot 7 \mathrm{H}_{2} \mathrm{O}\right.$ and $0.5 \mathrm{~g} \mathrm{CuSO}_{4} \cdot 5 \mathrm{H}_{2} \mathrm{O}$ in $100 \mathrm{~mL} \mathrm{H}_{2} \mathrm{O}$ ) and cultivated for 14 days at $25^{\circ} \mathrm{C}$ in the dark.

\subsection{Chemical Analyses}

The cultures were extracted with $3 \mathrm{~mL}$ acetonitrile/water/acetic acid (79/20/1) using a Vibracell VC130 sonicator (Sonics \& Materials, Inc., Newtown, CT, USA) with an amplitude of 100 for 10 seconds per sample subsequently by rotation at $180 \mathrm{rpm}$ for 1.5 hours. The extracts were spun for two minutes at 12,000 rpm in $2 \mathrm{~mL}$ tubes and then transferred to $2 \mathrm{~mL}$ HPLC vials for analysis. The samples were analyzed on a dionex UltiMate 3000 UHPLC system (Dionex, Idstein, Germany) connected to a Thermo Vantage triple stage quadrupole mass spectrometer (Thermo Fisher Scientific, San José, CA, USA) with a heated electrospray ionization probe. $5 \mu \mathrm{L}$ were injected and separated on a Gemini C6-Phenyl $3 \mu \mathrm{m}$ 2-mm i.d. $\times$ 50-mm column (Phenomenex, Torrance, CA, USA) using a constant flow of a $0.4 \mathrm{~mL} / \mathrm{min}$ and gradient system consisting of $\mathrm{A}\left(\mathrm{H}_{2} \mathrm{O}\right.$ :acetic acid; $\left.99: 1\right)$ and $\mathrm{B}$ (MeCN: $\mathrm{H}_{2} \mathrm{O}$ :acetic acid; 89:10:1), both buffered with $5 \mathrm{mM}$ ammonium acetate. The gradient started at $0 \% \mathrm{~B}$ increasing to $100 \%$ over $10 \mathrm{~min}$, which was maintained for three minutes before reverting to $0 \% \mathrm{~B}$ in one minute and recalibrated for two minutes. The following ion source parameters were used for detection: spray voltage $(4.5 \mathrm{kV})$, vaporizer temperature $\left(350{ }^{\circ} \mathrm{C}\right)$, nitrogen sheath gas pressure (30 arbitrary units), nitrogen auxiliary gas pressure (10 arbitrary units), capilliary temperature $\left(270{ }^{\circ} \mathrm{C}\right)$. Argon was used as the collision gas and set to $1.5 \mathrm{mTorr}$. Collision energy and selected reaction monitoring (SRM) transitions were automatically optimized for moniliformin, fusarin $\mathrm{C}$, antibiotic $\mathrm{Y}$ and enniatin A, A1, B and B1, whereas aurofusarin, chlamydosporol and 2-AOD-3-ol was 
tuned from extract of $F$. avenaceum grown on yeast extract sucrose agar (Table 1). Ergosterol was measured at $280 \mathrm{~nm}$ on an Agilent 1200 LC system (Agilent Technologies, Waldbronn, Germany) equipped with a diode array detector. Five $\mu \mathrm{L}$ extract was injected and separated on a $100 \times 2.1 \mathrm{~mm}$ kinetex $2.6 \mu \mathrm{m}$ phenyl-hexyl (Phenomenex, Torrance, CA, USA) using a flow of $0.4 \mathrm{~mL} / \mathrm{min}$ with a linear water-acetonitrile gradient, where both eluents were buffered with $50 \mathrm{ppb}$ trifluoro acetic acid. The gradient started at 5\% acetonitrile and reached $100 \%$ in $10 \mathrm{~min}$, which was held for $2 \mathrm{~min}$.

\section{Conclusions}

The results showed that $F$. avenaceum strains IBT 8500 and IBT 40847 grew better on the three polysaccharides than the mono- and disaccharides, whereas strain IBT 5001 had slightly higher ergosterol levels when grown on mono- and disaccharides.

All three strains were able to grow and produce aurofusarin on all the tested carbon sources. Lactose resulted in the lowest production of aurofusarin, chlamydosporol, enniatin $\mathrm{B}$, moniliformin and fusarin $\mathrm{C}$ levels under the tested conditions, which can be due to reduced growth or direct or indirect effects on biosynthesis of secondary metabolites in $F$. avenaceum. Enniatin B and moniliformin were produced on most of the carbon sources although variation between the strains was observed. Antibiotic Y, 2-AOD-3-ol and chlamydosporol levels varied more between the different carbon sources in each strain, which could suggest that production of these secondary metabolites is under the influence of regulators which respond to carbon source.

\section{Acknowledgments}

This project was supported by grants from The Danish Research Council, Technology and Production (12-132415).

\section{Conflicts of Interest}

The authors declare no conflict of interest.

\section{References}

1. Sørensen, J.L.; Nielsen, K.F.; Thrane, U. Analysis of moniliformin in maize plants using hydrophilic interaction chromatography. J. Agric. Food Chem. 2007, 55, 9764-9768.

2. Kosiak, B.; Torp, M.; Skjerve, E.; Thrane, U. The prevalence and distribution of Fusarium species in Norwegian cereals: A survey. Acta Agric. Scand. Sect. B. Soil Plant Sci. 2003, 53, 168-176.

3. Andersen, B.; Thrane, U.; Svendsen, A.; Rasmussen, I.A. Associated field mycobiota on malt barley. Can. J. Bot. 1996, 74, 854-858.

4. Yli-Mattila, T.; Paavanen-Huhtala, S.; Parikka, P.; Konstantinova, P.; Gagkaeva, T.Y. Molecular and morphological diversity of Fusarium species in Finland and north-western Russia. Eur. J. Plant Pathol. 2004, 110, 573-585.

5. Turkington, T.K.; Clear, R.M.; Burnett, P.A.; Patrick, S.K.; Orr, D.D.; Xi, K. Fungal plant pathogens infecting barley and wheat seed from Alberta, 1995-1997. Can. J. Plant Pathol. 2002, 24, 302-308. 
6. Sørensen, J.L.; Phipps, R.K.; Nielsen, K.F.; Frank, J.; Schroers, H.J.; Thrane, U. Analysis of Fusarium avenaceum metabolites produced during wet apple core rot. J. Agric. Food Chem. 2009, 57, 1632-1639.

7. Peters, R.D.; Barasubiye, T.; Driscoll, J. Dry rot of rutabaga caused by Fusarium avenaceum. Hortscience 2007, 42, 737-739.

8. Peters, J.C.; Lees, A.K.; Cullen, D.W.; Sullivan, L.; Stroud, G.P.; Cunnington, A.C. Characterization of Fusarium spp. responsible for causing dry rot of potato in Great Britain. Plant Pathol. 2008, 57, 262-271.

9. Tseng, T.C.; Tu, J.C. Mycoflora and mycotoxins in adzuki and mung beans produced in Ontario, Canada. Microbios 1997, 90, 87-95.

10. Uhlig, S.; Jestoi, M.; Knutsen, A.K.; Heier, B.T. Multiple regression analysis as a tool for the identification of relations between semi-quantitative LC-MS data and cytotoxicity of extracts of the fungus Fusarium avenaceum (syn. F. arthrosporioides). Toxicon 2006, 48, 567-579.

11. Uhlig, S.; Jestoi, M.; Parikka, P. Fusarium avenaceum-The North European situation. Int. J. Food Microbiol. 2007, 119, 17-24.

12. Jestoi, M. Emerging Fusarium-mycotoxins fusaproliferin, beauvericin, enniatins, and moniliformin-A review. Crit. Rev. Food Sci. Nutr. 2008, 48, 21-49.

13. Jestoi, M.; Rokka, M.; Yli-Mattila, T.; Parikka, P.; Rizzo, A.; Peltonen, K. Presence and concentrations of the Fusarium-related mycotoxins beauvericin, enniatins and moniliformin in Finnish grain samples. Food Addit. Contam. 2004, 21, 794-802.

14. Keller, N.P.; Hohn, T.M. Metabolic pathway gene clusters in filamentous fungi. Fungal Genet. Biol. 1997, 21, 17-29.

15. Brakhage, A.A. Regulation of fungal secondary metabolism. Nat. Rev. Microbiol. 2013, 11, 21-32.

16. Gardiner, D.M.; Kazan, K.; Manners, J.M. Nutrient profiling reveals potent inducers of trichothecene biosynthesis in Fusarium graminearum. Fungal Genet. Biol. 2009, 46, 604-613.

17. Jiao, F.; Kawakami, A.; Nakajima, T. Effects of different carbon sources on trichothecene production and Tri gene expression by Fusarium graminearum in liquid culture. FEMS Microbiol. Lett. 2008, 285, 212-219.

18. Sørensen, J.L.; Akk, E.; Thrane, U.; Giese, H.; Sondergaard, T.E. Production of fusarielins by Fusarium. Int. J. Food Microbiol. 2013, 160, 206-211.

19. Min, K.; Shin, Y.; Son, H.; Lee, J.; Kim, J.-C.; Choi, G.J.; Lee, Y.-W. Functional analyses of the nitrogen regulatory gene areA in Gibberella zeae. FEMS Microbiol. Lett. 2012, 334, 66-73.

20. Linnemannstöns, P.; Schulte, J.; Prado, M.D.; Proctor, R.H.; Avalos, J.; Tudzynski, B. The polyketide synthase gene pks4 from Gibberella fujikuroi encodes a key enzyme in the blosynthesis of the red pigment bikaverin. Fungal Genet. Biol. 2002, 37, 134-148.

21. Mihlan, M.; Homann, V.; Liu, T.W.D.; Tudzynski, B. AREA directly mediates nitrogen regulation of gibberellin biosynthesis in Gibberella fujikuroi, but its activity is not affected by NMR. Mol. Microbiol. 2003, 47, 975-991.

22. Macios, M.; Caddick, M.X.; Weglenski, P.; Scazzocchio, C.; Dzikowska, A. The GATA factors AREA and AREB together with the co-repressor NMRA, negatively regulate arginine catabolism in Aspergillus nidulans in response to nitrogen and carbon source. Fungal Genet. Biol. 2012, 49, 189-198. 
23. Brakhage, A.A. Molecular regulation of beta-lactam biosynthesis in filamentous fungi. Microbiol. Mol. Biol. Rev. 1998, 62, 547-585.

24. Kokkonen, M.; Ojala, L.; Parikka, P.; Jestoi, M. Mycotoxin production of selected Fusarium species at different culture conditions. Int. J. Food Microbiol. 2010, 143, 17-25.

25. Kleigrewe, K.; Sohnel, A.C.; Humpf, H.U. A new high-performance liquid chromatography-tandem mass spectrometry method based on dispersive solid phase extraction for the determination of the mycotoxin fusarin C in corn ears and processed corn samples. J. Agric. Food Chem. 2011, 59, 10470-10476.

26. Solfrizzo, M.; Visconti, A.; Savard, M.E.; Blackwell, B.A.; Nelson, P.E. Isolation and characterization of new chlamydosporol related metabolites of Fusarium chlamydosporum and Fusarium tricinctum. Mycopathologia 1994, 127, 95-101.

27. Strauss, J.; Horvath, H.K.; Abdallah, B.M.; Kindermann, J.; Mach, R.L.; Kubicek, C.P. The function of CreA, the carbon catabolite repressor of Aspergillus nidulans, is regulated at the transcriptional and post-transcriptional level. Mol. Microbiol. 1999, 32, 169-178.

28. Flipphi, M.; van de Vondervoort, P.J.I.; Ruijter, G.J.G.; Visser, J.; Arst, H.N.; Felenbok, A. Onset of carbon catabolite repression in Aspergillus nidulans-Parallel involvement of hexokinase and glucokinase in sugar signaling. J. Biol. Chem. 2003, 278, 11849-11857.

29. Hajjaj, H.; Niederberger, P.; Duboc, P. Lovastatin biosynthesis by Aspergillus terreus in a chemically defined medium. Appl. Environ. Microbiol. 2001, 67, 2596-2602.

30. Sondergaard, T.E.; Hansen, F.T.; Purup, S.; Nielsen, A.K.; Giese, H.; Sørensen, J.L. Fusarin C acts like an estrogenic agonist and stimulates breast cancer cells in vitro. Toxicol. Lett. 2011, 205, $116-121$.

31. Yoder, W.T.; Christianson, L.M. Species-specific primers resolve members of Fusarium section Fusarium-Taxonomic status of the edible "Quorn" fungus reevaluated. Fungal Genet. Biol. 1998, 23, 68-80.

(C) 2013 by the authors; licensee MDPI, Basel, Switzerland. This article is an open access article distributed under the terms and conditions of the Creative Commons Attribution license (http://creativecommons.org/licenses/by/3.0/). 